\title{
Overall Survival and Health Care Costs of Medicare Patients with Previously Treated Chronic Lymphocytic Leukemia
}

\author{
Carolina Reyes ${ }^{1}$, Genevieve Gauthier ${ }^{2}$, Sherry Shi ${ }^{2}$, Annie Guerin ${ }^{2 *}$ \\ ${ }^{1}$ Genentech Inc., South San Francisco, CA, USA \\ ${ }^{2}$ Analysis Group Inc., Montreal, Canada \\ Email: *annie.guerin@analysisgroup.com
}

How to cite this paper: Reyes, C., Gautheir, G., Shi, S. and Guerin, A. (2018) Overall Survival and Health Care Costs of Medicare Patients with Previously Treated Chronic Lymphocytic Leukemia. Journal of Cancer Therapy, 9, 576-587. https://doi.org/10.4236/jct.2018.97049

Received: June 25, 2018

Accepted: July 20, 2018

Published: July 23, 2018

Copyright (๑) 2018 by authors and Scientific Research Publishing Inc. This work is licensed under the Creative Commons Attribution International License (CC BY 4.0).

http://creativecommons.org/licenses/by/4.0/ (c) (i) Open Access

\begin{abstract}
Background: Bendamustine-based regimens are often used in the management of patients with chronic lymphocytic leukemia (CLL) but few studies have analyzed the comorbidity- and/or adverse event (CAE)-related healthcare costs in patients receiving these regimens in a real-world setting. Aims: To describe all-cause and CAE-related healthcare costs in relapse/refractory $(\mathrm{R} / \mathrm{R})$ elderly patients with CLL treated with bendamustine-based regimens in a real-world setting. Methods: Adult patients with R/R CLL who received bendamustine-based regimens on/after January 2010 were selected from the Medicare Limited Data Set (LDS) 5\% Standard Analytic Files. Selected patients were classified into cohorts based on the two most prevalent bendamustine-based regimens observed (index treatment): 1) bendamustine + rituximab (BR cohort) and 2) bendamustine monotherapy (B-mono cohort). For each cohort, all-cause and CAE-related healthcare costs, while on treatment, were reported per-patient-per-month (PPPM). Overall survival (OS) rates following initiation of the index treatment were described using age- and gender-adjusted Kaplan-Meier curves. Results: A total of 275 patients were included in the BR cohort and 100 patients in the B-mono cohort. Most patients were male and the mean age was approximately 75 years old. During treatment, total all-cause healthcare costs were $\$ 14,520$ PPPM for the BR cohort and \$13,125 PPPM for the B-mono cohort-outpatient costs (mainly driven by CLL-drug costs) represented $86.1 \%$ of the total all-cause healthcare costs for the BR cohort and $69.8 \%$ for the B-mono cohort. CAE costs accounted for $58.3 \%$ of the total all-cause healthcare costs for the BR cohort and $66.9 \%$ for the B-mono cohort. Median OS was 35 months in the BR cohort and 21 months in the B-mono cohort. Conclusion: In this population of elderly patients with $\mathrm{R} / \mathrm{R}$ CLL treated with bendamustine-based regimens,
\end{abstract}


CAEs were common and translated into important medical costs. Median OS was also relatively short suggesting an unmet medical need.

\section{Keywords}

Chronic Lymphocytic Leukemia, Relapse/Refractory, Bendamustine, Economic Burden, Overall Survival, Adverse Events

\section{Introduction}

Chronic lymphocytic leukemia (CLL) is a type of lymphoproliferative neoplasm characterized by progressive accumulation of mature but functionally incompetent B-lymphocytes in the blood, bone marrow, and in later stages of the disease, lymph nodes, liver, and spleen [1] [2]. It is the most common form of leukemia in adults in western countries. The incidence rate rises with age with median age at diagnosis reported to be between 70 and 72 years [3].

Most patients with CLL typically experience an indolent course of their disease and remain asymptomatic for many years [4]. However, the clinical course of CLL is highly variable and given most patients' advanced age and high comorbidity burden at time of diagnosis, optimal treatment decisions must consider multiple aspects of a patient's profile, including age, presence of comorbidities, genomic changes, and mutational status (e.g. 17p deletion/TP53 mutation) [1] Despite advances in treatment for patients with CLL, there are currently no curative treatments; refractory disease (treatment failure/disease progression within 6 months of the last dose of therapy) and disease relapse (evidence of disease progression 6 months or more after achievement of complete response/partial response) after initial treatment is common [5] [6]. Patients with relapsed or refractory $(\mathrm{R} / \mathrm{R})$ disease generally have poor outcomes and their treatment remains highly challenging [7]. Until recently, chemotherapy and chemo-immunotherapy were the standard of care for patients with R/R CLL. Since 2014, new oral targeted therapies for R/R CLL have entered the market [7] [8] [9]. These new agents are currently evaluated in clinical trials with or against a bendamustine-based regimen, which is commonly used in real-world practice [10] [11].

However, to date there is no point of reference for real-world outcomes of patients with CLL treated with bendamustine-based regimens. The aim of this study was to describe healthcare costs, including comorbidity- and/or adverse event (CAE)-related healthcare costs, and overall survival (OS) rates in an elderly population of patients with R/R CLL treated with a bendamustine-based regimen.

\section{Methods}

\subsection{Data Source}

A retrospective cross-sectional study design was used. Given that the population of patients with CLL is primarily composed of elderly patients and that OS was 
an outcome of interest in this study, data were extracted from the Medicare Limited Data Set (LDS) 5\% Standard Analytic Files (data availability: 1999-2014) [12]. The database includes information on beneficiaries' Medicare enrollment, medical resources used (e.g., hospitalizations, emergency room visits), and associated payments made by Medicare to providers, as well as survival and mortality data. The data are de-identified and comply with the confidentiality requirements of the Health Insurance Portability and Accountability Act.

\subsection{Sample Selection}

Patients with CLL (International Classification of Diseases, Ninth Edition, Clinical Modification [ICD-9-CM] code 204.1x) who received a bendamustine-based regimen in a medical setting in second or later lines of therapy on/after January 2010 were identified. The index date was defined as the initiation date for the first bendamustine-based regimen. Selected patients were required to be continuously enrolled in their healthcare plan for $\geq 6$ months before the index date and $\geq 3$ months after the index date (unless the patients died during the first 3 months after the index date, in which case no minimum follow-up was required). Patients in this study were required to be $\geq 65$ years of age as of the index date.

The sample of patients was limited to patients with an indicator of R/R CLL before the initiation of the bendamustine-based regimen. As laboratory test results and clinical information are not available in claims databases, an empirical algorithm based on treatment patterns was used to identify patients with $R / R$ CLL. More specifically, $R / R$ patients were identified based on the following treatment changes 1) a treatment add-on, 2) the initiation or re-initiation of a CLL treatment after a discontinuation (treatment interruption of $\geq 60$ consecutive days), or 3 ) a treatment switch that was not preceded by claims for a medical service, in an inpatient or emergency room setting, with a diagnosis for a CAE.

\subsection{Study Cohorts}

Patient cohorts were determined based on the treatment initiated on the index date (index treatment). The two most common bendamustine-based regimens were selected for analysis: 1) combination of bendamustine and rituximab (BR cohort) and 2) bendamustine in monotherapy (B-mono cohort) [10]. Figure 1 shows an example for the BR cohort.

\subsection{Outcomes and Statistical Analyses}

\section{Healthcare costs}

All-cause healthcare costs, including inpatient, emergency department, and outpatient costs were measured during the line of therapy for the index treatment, i.e., between the index date and the date of the first treatment change (switch, drop, add-on, or discontinuation), the end of continuous healthcare 


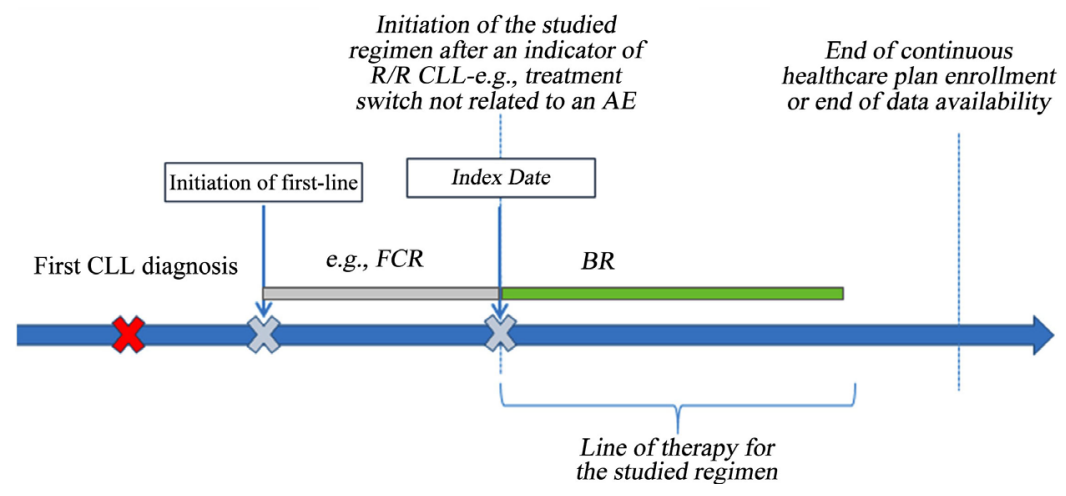

Figure 1. Study design. Abbreviations: AE: adverse event; BR: bendamustine and rituximab; CLL: chronic lymphocytic leukemia; FCR: fludarabine, cyclophosphamide, and rituximab; R/R: relapse/refractory.

plan enrollment, the end of data availability, or the date of a patient's death, whichever occurred first.

Total CLL-drug cost and total CAE-related cost were also reported. The total CLL-drug cost was defined as the sum of the costs associated with CLL drug and administration in a medical setting (including inpatient, outpatient, or emergency department). The total CAE-related cost was defined as the sum of the costs for claims with inpatient, emergency department, and outpatient services including an ICD-9-CM diagnosis code for one of the studied CAEs-the list of studied CAEs was created based on a review of the adverse events reported in product labels and clinical trials. As patients could have both a procedure for CLL treatment administration and a diagnosis code for one of the selected CAEs in the same claim, and there is no information in claims data to distinguish the portion of the costs associated with treatment administration versus services related to CAEs, CLL drug cost and CAE-related cost were reported as non-mutually exclusive cost categories.

Healthcare costs were adjusted for inflation using the consumer price index for medical components (2016 US Dollars) and reflected the costs reimbursed by Medicare.

Healthcare costs were described for each cohort using mean, median, and standard deviation and reported per-patient-per-month (PPPM) as the duration of the line of therapy for the index treatment varied across patients. This study was descriptive; no statistical comparison was conducted.

\section{Overall survival}

OS rates were measured between the index date and the date of death, end of healthcare plan enrollment or end of data availability, whichever occurred first. OS rates were presented graphically as age- and gender-adjusted Kaplan-Meier curves. OS rates were reported separately for the two study cohorts. No statistical comparison was conducted.

\section{Results}

A total of 275 patients were included in the BR cohort and 100 patients in the 
B-mono cohort. Mean age was approximately 75 years in both cohorts, $61.8 \%$ of patients in the BR cohort and $65.0 \%$ of the patients in the B-mono cohort were men. During the 6 months prior to the index date, patients in both cohorts presented a high comorbidity burden. The mean Charlson Comorbidity Index (CCI) was 3.5 in the BR cohort and 3.7 in the B-mono cohort. Over half of the patients in both cohorts had a diagnosis for hypertension (BR cohort $=57.1 \%$; B-mono cohort $=54.0 \%$ ), approximately $30 \%$ of patients had a diagnosis of diabetes $(\mathrm{BR}$ cohort $=29.5 \%$; B-mono cohort $=27.0 \%$ ) and coagulopathy (BR cohort $=28.7 \%$; B-mono cohort $=26.0 \%$ ), and over $20 \%$ of patients had a diagnosis for cardiac arrhythmias (BR cohort $=24.7 \%$; B-mono cohort $=21.0 \%)$ and solid tumor $($ BR cohort $=20.7 \%$; B-mono cohort $=20.0 \%)($ Table 1$)$.

The mean follow-up period after the index date was 24 months for the BR cohort and 22 months for the B-mono cohort. The average duration of the line of therapy for the index treatment was 121 days (median $=116$ days) in the BR cohort and 75 days (median $=56$ days) in the B-mono cohort. Table 2 describes the proportion of patients with each of the most commonly reported CAEs during the line of therapy for the index treatment. Blood and lymphatic disorders (e.g., anemia, neutropenia, and thrombocytopenia) were the most common CAEs observed in both the BR (73.1\%) and B-mono (67.0\%) cohorts, followed by gastrointestinal disorders (58.9\% in the BR cohort, and $48.0 \%$ in the B-mono cohort) and general disorder (50.5\% in the BR cohort, and $47.0 \%$ in the B-mono cohort).

The total all-cause healthcare cost during the line of therapy for the index treatment was high in both cohorts: mean of \$14,520 PPPM in the BR cohort and $\$ 13,125$ PPPM in the B-mono cohort. Outpatient cost, mainly driven by CLL-drug cost, represented $86.1 \%$ of the cost in the BR cohort and $69.8 \%$ of the cost in the B-mono cohort. Total CAE-related cost (any CAE events) accounted for over half of the total all-cause healthcare cost; $58.3 \%$ for the BR cohort and $66.9 \%$ for the B-mono cohort (Table 3) - mean of $\$ 8462$ PPPM for the BR cohort and $\$ 8783$ for the B-mono cohort.

The median OS following the index date was 35 months in the BR cohort and 21 months in the B-mono cohort (Figure 2).

\section{Discussion}

Since 2014, new oral targeted therapies have been approved for treatment of patients with R/R CLL. These new agents are currently evaluated in clinical trials with or compared to bendamustine-based regimens. However, real-world data on outcomes in patients with R/R CLL receiving bendamustine-based regimens are lacking. This study provided evidence on all-cause and CAE-related costs and OS in an elderly population of patients with R/R CLL treated with bendamustine-based regimens in a real-world setting.

Results from the current study showed that the total healthcare costs during therapy were \$14,520 PPPM for the BR cohort and \$13,125 PPPM for the B-mono cohort. Outpatient cost, mainly driven by CLL-drug cost, represented 
the largest cost component. Results also showed that CAE-related healthcare costs accounted for $58 \%$ of total healthcare costs in the BR cohort and $67 \%$ in the $\mathrm{B}$-mono cohort. This finding is not surprising given the profile of this patient population, i.e., $\mathrm{R} / \mathrm{R}$ CLL patients in their late seventies with the presence of a relatively high comorbidity burden at the time of the initiation of the studied bendamustine-based regimens.

Table 1. Patient characteristics.

\begin{tabular}{|c|c|c|}
\hline & $\mathrm{BR}$ & B-mono \\
\hline & $\mathrm{n}=275$ & $\mathrm{n}=100$ \\
\hline Age, mean $\pm \mathrm{SD}$ [median] & $75.6 \pm 6.5[75.0]$ & $75.4 \pm 7.0[74.0]$ \\
\hline $65-69$ years, n (\%) & $42(15.3)$ & $18(18.0)$ \\
\hline $70-74$ years, $\mathrm{n}(\%)$ & $70(25.5)$ & $25(25.0)$ \\
\hline 75 - 79 years, $n(\%)$ & $73(26.5)$ & $27(27.0)$ \\
\hline$\geq 80$ years, $\mathrm{n}(\%)$ & $90(32.7)$ & $30(30.0)$ \\
\hline Male, n (\%) & $170(61.8)$ & $65(65.0)$ \\
\hline \multicolumn{3}{|l|}{ Region of residence, $n(\%)$} \\
\hline South & $89(32.4)$ & $40(40.0)$ \\
\hline North central & $46(16.7)$ & $22(22.0)$ \\
\hline West & $35(12.7)$ & $9(9.0)$ \\
\hline North east & $69(25.1)$ & $23(23.0)$ \\
\hline Unknown & $36(13.1)$ & $6(6.0)$ \\
\hline \multicolumn{3}{|l|}{ Year of index date, $n(\%)$} \\
\hline 2010 & $69(25.1)$ & $53(53.0)$ \\
\hline 2011 & $63(22.9)$ & $20(20.0)$ \\
\hline 2012 & $60(21.8)$ & $15(15.0)$ \\
\hline 2013 & $56(20.4)$ & $10(10.0)$ \\
\hline 2014 & $27(9.8)$ & $2(2.0)$ \\
\hline $\begin{array}{l}\text { Months from first CLL diagnosis to index date, } \\
\text { mean } \pm \text { SD [median] }\end{array}$ & $61.31 \pm 42.02[54.64]$ & $61.78 \pm 42.44[60.58]$ \\
\hline $\begin{array}{l}\text { Charlson comorbidity index (CCI), } \\
\text { mean } \pm \text { SD [median] }\end{array}$ & $3.53 \pm 1.82[3.00]$ & $3.66 \pm 1.87[3.00]$ \\
\hline \multicolumn{3}{|l|}{$\begin{array}{l}\text { Six most common physical and mental } \\
\text { comorbidities }\end{array}$} \\
\hline Hypertension & $157(57.1)$ & $54(54.0)$ \\
\hline Lymphoma & $134(48.7)$ & $53(53.0)$ \\
\hline Diabetes & $81(29.5)$ & $27(27.0)$ \\
\hline Coagulopathy & $79(28.7)$ & $26(26.0)$ \\
\hline Cardiac arrhythmias & $68(24.7)$ & $21(21.0)$ \\
\hline Solid tumor & $57(20.7)$ & $20(20.0)$ \\
\hline
\end{tabular}

Abbreviations: B-mono: bendamustine in monotherapy; BR: bendamustine and rituximab; CCI: Charlson comorbidity index; CLL: chronic lymphocytic leukemia; SD: standard deviation. 
Table 2. Rates of most commonly reported CAEs during the line of therapy.

\begin{tabular}{|c|c|c|}
\hline Patients with CAE, n (\%) & BR & B-mono \\
\hline & $\mathrm{n}=275$ & $\mathrm{n}=100$ \\
\hline $\begin{array}{l}\text { Duration of the line of therapy (days), } \\
\text { mean [median] }\end{array}$ & $121[116]$ & $75[56]$ \\
\hline Blood and lymphatic system disorders & $201(73.1)$ & $67(67.0)$ \\
\hline Anemia (deficiency and hemolytic) & $73(26.5)$ & $35(35.0)$ \\
\hline Neutropenia and febrile neutropenia & $112(40.7)$ & $37(37.0)$ \\
\hline Lymphadenopathy & $62(22.5)$ & $18(18.0)$ \\
\hline Thrombocytopenia (including autoimmune) & $78(28.4)$ & $25(25.0)$ \\
\hline Cardiac disorders & $75(27.3)$ & $21(21.0)$ \\
\hline $\begin{array}{l}\text { Arrhythmia (including atrial fibrillation and } \\
\text { flutter) }\end{array}$ & $58(21.1)$ & $12(12.0)$ \\
\hline Cardiac failure congestive & $35(12.7)$ & $11(11.0)$ \\
\hline Endocrine disorders, i.e., hypothyroidism & $47(17.1)$ & $6(6.0)$ \\
\hline Gastrointestinal disorders & $162(58.9)$ & $48(48.0)$ \\
\hline Nausea/vomiting & $97(35.3)$ & $34(34.0)$ \\
\hline General disorders & $139(50.5)$ & $47(47.0)$ \\
\hline Chills/pyrexia (with and without fever) & $50(18.2)$ & $21(21.0)$ \\
\hline Malaise and fatigue & $78(28.4)$ & $25(25.0)$ \\
\hline Hepatobiliary disorders & $1(0.4)$ & $0(0.0)$ \\
\hline $\begin{array}{l}\text { Injury, poisoning and procedural } \\
\text { complications }\end{array}$ & $34(12.4)$ & $21(21.0)$ \\
\hline $\begin{array}{l}\text { Allergic reaction }+ \text { application/administration } \\
\text { site reaction }\end{array}$ & $30(10.9)$ & $20(20.0)$ \\
\hline Metabolism and nutrition disorders & $114(41.5)$ & $28(28.0)$ \\
\hline Dehydration & $54(19.6)$ & $18(18.0)$ \\
\hline Hypokalaemia & $25(9.1)$ & $4(4.0)$ \\
\hline $\begin{array}{c}\text { Musculoskeletal and connective tissue } \\
\text { disorders }\end{array}$ & $69(25.1)$ & $17(17.0)$ \\
\hline Neoplasms & $97(35.3)$ & $24(24.0)$ \\
\hline Nervous system disorder & $50(18.2)$ & $10(10.0)$ \\
\hline Renal and urinary disorders & $71(25.8)$ & $15(15.0)$ \\
\hline Psychiatric disorders & $35(12.7)$ & $12(12.0)$ \\
\hline $\begin{array}{c}\text { Respiratory, thoracic and mediastinal } \\
\text { disorders }\end{array}$ & $136(49.5)$ & $43(43.0)$ \\
\hline Cough & $60(21.8)$ & $19(19.0)$ \\
\hline Dyspnea & $69(25.1)$ & $20(20.0)$ \\
\hline Skin and subcutaneous tissue disorders & $26(9.5)$ & $5(5.0)$ \\
\hline Vascular disorders & $156(56.7)$ & $39(39.0)$ \\
\hline Hypertension & $134(48.7)$ & $31(31.0)$ \\
\hline Infections & $141(51.3)$ & $39(39.0)$ \\
\hline
\end{tabular}

Abbreviations: B-mono: bendamustine in monotherapy; BR: bendamustine and rituximab; CAE: comorbidity and/or adverse event. 
Table 3. Healthcare costs PPPM during the line of therapy.

\begin{tabular}{ccc}
\hline Healthcare cost PPPM, mean \pm SD [median] & BR & B-mono \\
\hline Total all-cause healthcare costs & $\mathbf{n}=\mathbf{2 7 5}$ & $\mathbf{n}=100$ \\
Inpatient & $14,520 \pm 5760[13,755]$ & $13,125 \pm 9439[10,607]$ \\
Emergency department & $1922 \pm 4789[0]$ & $3720 \pm 7745[0]$ \\
Other outpatient & $96 \pm 331[0]$ & $243 \pm 836[0]$ \\
Total CLL drug costs ${ }^{\mathbf{a}}$ & $12,501 \pm 3741[12,310]$ & $9163 \pm 4788[8627]$ \\
Total CAE-related healthcare costs ${ }^{\mathbf{a}}$ & $\mathbf{1 0 , 5 6 8 \pm 3 5 0 6 [ 1 0 , 4 4 7 ]}$ & $\mathbf{7 0 5 5} \pm \mathbf{3 6 7 2 [ 6 6 4 3 ]}$ \\
Inpatient & $1922 \pm 4789[0]$ & $3703 \pm 7751[0]$ \\
Emergency department & $91 \pm 321[0]$ & $120 \pm 411[0]$ \\
Other outpatient & $6449 \pm 5293[6209]$ & $4961 \pm 5288[3421]$ \\
\hline
\end{tabular}

Abbreviations: B-mono: bendamustine in monotherapy; BR: bendamustine and rituximab; CAE: comorbidity and/or adverse event; CLL: chronic lymphocytic leukemia; PPPM: per-patient-per-month; SD: standard deviation. ${ }^{a}$ CLL drug cost and CAE-related healthcare cost categories are not mutually exclusive.

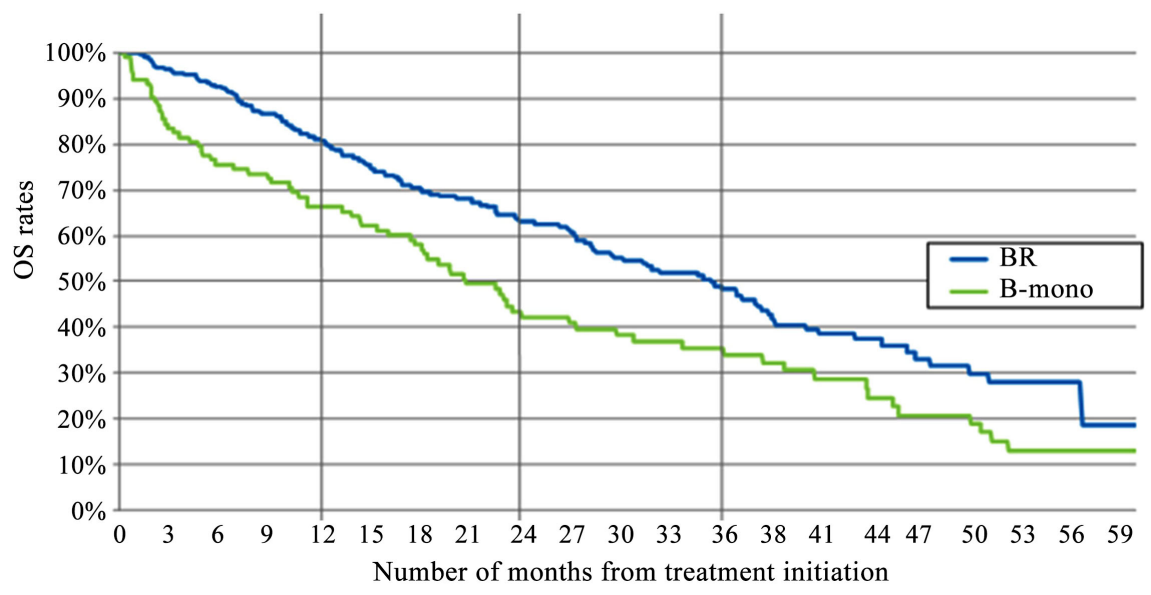

Figure 2. Overall survival rates. Abbreviations: B-mono: bendamustine in monotherapy; BR: bendamustine and rituximab; OS: overall survival.

To the best of our knowledge, this is the first study to assess all-cause and CAE-related healthcare costs in a sample of R/R CLL patients treated with bendamustine-based regimens. One prior study has assessed healthcare costs in patients with R/R CLL in the US (not specific to BR or B-mono therapy) and estimated mean total healthcare costs of $\$ 14,920$ PPPM during the first month following R/R CLL, mean costs above $\$ 7000$ PPPM for the next 3 months, and costs ranging between $\$ 4231$ and $\$ 5992$ PPPM over the subsequent months [13]. The higher costs in the current study ( $\$ 14,520$ PPPM for the BR cohort, $\$ 13,125$ PPPM for the B-mono cohort) are not surprising given that the sample of patients comprises older patients (median age 75 versus 68 years) with a high comorbidity burden, that costs were measured while on treatment (versus intent to treat design), and that the current study was specific to bendamustine-based 
regimens while the prior study included a mix of treatments-different treatments may have very different cost profiles and the choice of treatment may be endogenous to patient profiles. The total healthcare cost estimated in the current study is also higher than the costs estimated from another recent US study conducted in patients with CLL (not specific to R/R CLL), in which the authors estimated a total healthcare cost (including amount reimbursed by the insurance plan and patients out of pocket expenses) of $\$ 101,445$ over a 9-month study period (translating into $\$ 11,272$ PPPM) for patients treated with BR [14]. This difference is again not surprising as patients with R/R CLL may be more complex to treat/manage, which is likely to translate into more intensive medical services and higher healthcare costs.

In the current study, the median OS was relatively short, with 35 months in the BR cohort and 21 months in the B-mono cohort, suggesting an unmet medical need. Comparisons of these results with existing literature are limited. To the best of our knowledge, this study is the first to assess OS in patients who were treated with bendamustine-based regimens in a real-world setting. In addition, although OS has been reported in clinical trials, results from the current study cannot be directly compared with those from clinical trials given the fundamental differences in study design and patient population. For example, elderly patients with a high comorbidity burden and poor performance status are often underrepresented in clinical trials, although they represent the bulk of the CLL population treated in real-world settings [15] [16].

The management of patients with $\mathrm{R} / \mathrm{R}$ CLL can be particularly challenging. Treatments need to consider multiple aspects of a patient's profile, including the age of the patient, presence of significant comorbidities, and genetic/mutation status. In this population of elderly patients, results suggest that the management of CLL can be complicated by the presence of comorbidities and the risk of developing CAEs, which can limit the treatment options and translate into important medical costs. These results suggest a need for a multidisciplinary approach in the management of CLL.

The current study is subject to limitations inherent in the use of claims databases. First, the claims databases only record diagnostic and procedural codes and do not include information on disease severity which may impact the choice of treatment regimens and outcomes. Second, due to the unavailability of laboratory test results and clinical information in claims databases, an empirical algorithm based on treatment patterns was used to identify patients with R/R CLL. Third, in 2014, novel oral agents were approved by the Food and Drug Administration for the treatment of patients with CLL. However, since the Medicare databases do not include Part D claims (i.e., information on prescription drugs), it was not possible to identify and exclude these patients from the analysis. Additional studies would be needed to assess the healthcare costs in patients with $\mathrm{R} / \mathrm{R}$ CLL treated with novel oral agents in a real-world setting. Fourth, claims databases do not include information on the cause of concomitant comorbid condi- 
tions. Accordingly, this study assessed the association of costs and CAE rather than the causation between the CAE and treatments. Fifth, in some cases, CLL drug costs and CAE-related costs could not be distinguished, as both a procedure for CLL treatment administration and a diagnosis code for one of the selected CAEs could be observed in the same claim. Costs for these specific components should be interpreted in light of this limitation. Finally, healthcare costs reported in this study reflect the medical costs reimbursed by Medicare. Therefore, the generalizability of the findings is limited to a population of elderly adult patients with CLL insured by Medicare, and may not be representative of the costs reimbursed for CLL patients insured by private payers.

\section{Conclusion}

In this population of elderly patients with $\mathrm{R} / \mathrm{R}$ CLL treated with bendamustine-based regimens, CAEs were common and translated into important medical costs. Median OS was also relatively short suggesting an unmet medical need. Management of patients with R/R CLL can be particularly challenging and must consider multiple aspects of a patient's profile including age, presence of comorbidities, and genetic/mutation profile. These complexities point to a need for a multidisciplinary approach in the management of patients with R/R CLL. However, the treatment outlook of CLL remains dynamic and rapidly developing with combination regimens using targeted novel agents holding considerable promise.

\section{Acknowledgements}

Carolina Reyes is an employee of Genentech Inc. and may own stocks/stock options. Genevieve Gauthier, Sherry Shi, and Annie Guerin are employees of Analysis Group Inc., which has received consultancy fees from Genentech Inc.

Venetoclax is being developed in collaboration between Genentech and AbbVie. Genentech and AbbVie provided financial support for the study. Genentech participated in the design, study conduct, analysis and interpretation of data, as well as the writing, review, and approval of the manuscript. Abbvie participated in the review and approval of the manuscript.

Medical writing assistance was provided by Sara Kaffashian, an employee of Analysis Group Inc.

\section{References}

[1] Kipps, T.J., Stevenson, F.K., Wu, C.J., Croce, C.M., Packham, G., Wierda, W.G., et al. (2017) Chronic Lymphocytic Leukaemia. Nature Reviews Disease Primers volume, 3, Article No. 16096. https://doi.org/10.1038/nrdp.2016.96

[2] Zhang, S. and Kipps, T.J. (2014) The Pathogenesis of Chronic Lymphocytic Leukemia. Annual Review of Pathology: Mechanisms of Disease, 9, 103-118. https://doi.org/10.1146/annurev-pathol-020712-163955

[3] Nabhan, C. and Rosen, S.T. (2014) Chronic Lymphocytic Leukemia: A Clinical Re- 
view. JAMA, 312, 2265-2276. https://doi.org/10.1001/jama.2014.14553

[4] Hallek, M. (2017) Chronic Lymphocytic Leukemia: 2017 Update on Diagnosis, Risk Stratification, and Treatment. American Journal of Hematology, 92, 946-965. https://doi.org/10.1002/ajh.24826

[5] Al-Sawaf, O., Cramer, P., Goede, V., Hallek, M. and Pflug, N. (2017) Bendamustine and Its Role in the Treatment of Unfit Patients with Chronic Lymphocytic Leukaemia: A Perspective Review. Therapeutic Advances in Hematology, 8, 197-205. https://doi.org/10.1177/2040620717699365

[6] Hallek, M., Cheson, B.D., Catovsky, D., Caligaris-Cappio, F., Dighiero, G., Dohner, H., et al. (2008) Guidelines for the Diagnosis and Treatment of Chronic Lymphocytic Leukemia: A Report from the International Workshop on Chronic Lymphocytic Leukemia Updating the National Cancer Institute-Working Group 1996 Guidelines. Blood, 111, 5446-5456. https://doi.org/10.1182/blood-2007-06-093906

[7] Chao, M.P. (2013) Treatment Challenges in the Management of Relapsed or Refractory Non-Hodgkin's Lymphoma-Novel and Emerging Therapies. Cancer Management and Research, 5, 251-269. https://doi.org/10.2147/CMAR.S34273

[8] US Food and Drug Administration (2014) Zydelig (Idelalisib) Tablets, Drug Apporval Package.

http://www.accessdata.fda.gov/drugsatfda_docs/nda/2014/206545Orig1s000TOC.cfm

[9] US Food and Drug Administration (2015) Imbruvica (Ibrutinib) Capsules, Drug Approval Package.

https://www.accessdata.fda.gov/drugsatfda_docs/nda/2014/205552Orig2s000TOC.cfm

[10] National Comprehensive Cancer Network (NCCN) (2018) Clinical Practice Guidelines in Oncology-Chronic Lymphocytic Leukemia/Small Lymphocytic Lymphoma Version 5.2018.

https://www.nccn.org/professionals/physician_gls/pdf/cll_blocks.pdf

[11] ClinicalTrials.gov. (2013) A Study to Evaluate the Benefit of Venetoclax plus Rituximab Compared with Bendamustine plus Rituximab in Participants with Relapsed or Refractory Chronic Lymphocytic Leukemia (CLL) (MURANO). National Library of Medicine, Bethesda. https://www.clinicaltrials.gov/ct2/show/NCT02005471

[12] Clark, M.A., Arnold, S.V., Duhay, F.G., Thompson, A.K., Keyes, M.J., Svensson, L.G., et al. (2012) Five-Year Clinical and Economic Outcomes among Patients with Medically Managed Severe Aortic Stenosis: Results from a Medicare Claims Analysis. Circulation: Cardiovascular Quality and Outcomes, 5, 697-704.

[13] Guerin, A., Ray, S., Gauthier, G., Hsu, L.I., Zhdanava, M., Heroux, J., et al. (2015) Economic Burden of Relapsed/Refractory Chronic Lymphocytic Leukemia. Journal of Clinical Oncology, 33.

http://ascopubs.org/doi/abs/10.1200/jco.2015.33.15_suppl.e18065

[14] Matasar, M.J., DaCosta Byfield, S., Blauer-Peterson, C., Montez, M., Reyes, C. and Masaquel, A. (2017) What Are the Health Care Costs for Chronic Lymphocytic Leukemia? Journal of Clinical Oncology, 35, 15.

[15] Robak, T., Hellmann, A., Kloczko, J., Loscertales, J., Lech-Maranda, E., Pagel, J.M., et al. (2017) Randomized Phase 2 Study of Otlertuzumab and Bendamustine versus Bendamustine in Patients with Relapsed Chronic Lymphocytic Leukaemia. British Journal of Haematology, 176, 618-628.

[16] Zelenetz, A.D., Barrientos, J.C., Brown, J.R., Coiffier, B., Delgado, J., Egyed, M., et al. (2017) Idelalisib or Placebo in Combination with Bendamustine and Rituximab in Patients with Relapsed or Refractory Chronic Lymphocytic Leukaemia: Interim 
Results from a Phase 3, Randomised, Double-Blind, Placebo-Controlled Trial. The Lancet Oncology, 18, 297-311. 